\title{
An Accurate Five Off-Step Points Implicit Block Method for Direct Solution of Fourth Order Differential Equations
}

\author{
Monday Kolawole Duromola \\ Department of Mathematical Sciences, Federal University of Technology, Akure, Nigeria \\ Email:mkduro@yahoo.com
}

Received 12 May 2016; accepted 19 June 2016; published 23 June 2016

Copyright (C) 2016 by author and OALib.

This work is licensed under the Creative Commons Attribution International License (CC BY). http://creativecommons.org/licenses/by/4.0/

(c) (i) Open Access

\begin{abstract}
In this article, my focus is the derivation, analysis and implementation of a new modified one-step implicit hybrid block method with five off-step points. The derived method is to solve directly initial value problems of fourth order ordinary differential equations. The approach for the derivation of the method is to interpolate the approximate power series solution to the problem and to collocate its fourth derivative at the grid and off-grid points to generate systems of linear equations for the determination of the unknown parameters. The derived method is tested for consistency, zero stability, convergence and absolute stability. Accuracy and usability of the method are determined with some test problems and the results obtained are found to be better in accuracy than some existing methods.
\end{abstract}

\section{Keywords}

Interpolation, Continuous Coefficients, Block Method, Numerical Integration, Fourth Order Ordinary Differential Equations

Subject Areas: Ordinary Differential Equation

\section{Introduction}

In sciences and engineering, mathematical models are developed to understand as well as to interpret physical phenomena, many of such phenomena, when modeled, often result into higher order ordinary differential equations of the form:

$$
y^{(i v)}=f\left(x, y, y^{\prime}, y^{\prime \prime}, y^{\prime \prime \prime}\right), \quad y(a)=\eta_{1}, y^{\prime}(a)=\eta_{2}, y^{\prime \prime}(a)=\eta_{3}, y^{\prime \prime \prime}(a)=\eta_{4}
$$

An old conventional way to solve (1) is the method of first reducing (1) to system of first order differential 
equation of the form:

$$
y^{\prime}=f(x, y), y(a)=\eta_{0}, f \in C[a, b], x, y \in \Re
$$

and to solve the resulting system of equations by any of the existing methods of solving first order ordinary differential equations. Literatures abounded in this old conventional method of solving problems of type (1) numerically are [1]-[3]. The drawbacks of this method include computational cumbersomeness and longer computer tine and space. In addition, [4] observes that these methods do not utilize additional information associated with a specific ordinary differential equation, such as oscillatory nature of the solution. To circumvent these drawbacks, many researchers have solved (1) directly; amongst these are [5]-[7] who develop blocked methods for numerical solution of fourth order ordinary differential equations. [8] develops linear multistep method for solution of fourth order ordinary differential equations whose implementation is Predictor-Corrector mode. Consequently, my motivation in this work is the success story of the adoption of single step method with five off-step points for direct numerical solution of fourth order ordinary differential equations which eliminate the use of predictors by providing sufficiently accurate simultaneous difference equations from a single continuous formula and its derivatives.

\section{Derivation of the Method}

We take our basis function to be a power series of the form:

$$
y(x)=\sum_{j=0}^{(r+s)-1} a_{j} \psi^{j}(x)
$$

The fourth derivative of (3) gives

$$
y^{(i v)}(x)=\sum_{j=0}^{(r+s)-1} j(j-1)(j-2)(j-3) a_{j} \psi^{j-4}(x)
$$

By putting (4) into (1) we have the differential system:

$$
\sum_{j=0}^{(r+s)-1} j(j-1)(j-2)(j-3) a_{j} \Psi^{j-4}(x)=f\left(x, y, y^{\prime}, y^{\prime \prime}, y^{\prime \prime \prime}\right)
$$

where $a_{j}$ 's are the parameters to be determining while $r+s$ denotes number of collation and interpolation points. By collocating (5) at the mesh points $x=x_{n+j}, j=0\left(\frac{1}{6}\right)$ and interpolating (3) at $x=x_{n+j}, j=\frac{1}{3}, \frac{1}{2}, \frac{2}{3}, \frac{5}{6}$ yields a system of equations:

$$
\begin{gathered}
\sum_{j=4}^{(r+s)-1} j(j-1)(j-2)(j-3) a_{j} \psi_{j-4}\left(x_{n+r}\right)=f_{n+r}, r=o, v_{i}, k \quad i=1,2, \cdots, m \\
\sum_{j=0}^{(r+s)-1} a_{j} \psi_{j}\left(x_{n+s}\right)=y_{n+s}, s=o, v_{i}, k \quad i=0,1,2, \cdots, m
\end{gathered}
$$

By putting these system of equations in the matrix form and then solved to obtain values of parameters $a_{j}$ 's, $j=0, \frac{1}{6}, \cdots$ which when substituted in (3), yields, after some manipulation, a hybrid linear method with continuous coefficients of the form:

$$
y(t)=\sum_{j=0}^{1} \alpha_{j}(t) y_{n+j}(t)+h^{4} \sum_{j=0}^{1} \beta_{j}(t) f_{n+j}(t)
$$

The coefficient of $\alpha_{j}(t)$ and $\beta_{j}(t)$ are

$$
\alpha_{0}(t)=\frac{1}{8}\left(2 t^{3}+t^{2}+5 t\right), \quad \alpha_{\frac{1}{3}}(t)=-\frac{1}{4}\left(8 t^{3}+22 t^{2}+10 t\right), \quad \alpha_{\frac{1}{2}}(t)=\frac{1}{2}\left(10 t^{3}+24 t^{2}+6 t\right)
$$




$$
\begin{gathered}
\alpha_{\frac{2}{3}}(t)=-\frac{1}{4}\left(32 t^{3}+18 t^{2}+7 t+3\right), \alpha_{\frac{5}{6}}(t)=\frac{1}{2}\left(3 t^{3}+4 t^{2}+t\right) \\
\beta_{0}(t)=\frac{1}{19595520}\left(12 t^{8}+t^{7}-4 t^{6}+2 t^{5}-4 t^{4}+t^{3}+4 t-6\right) \\
\beta_{\frac{1}{6}}(t)=\frac{1}{19595520}\left(258 t^{8}+496 t^{7}-315 t^{6}+998 t^{5}+945 t^{4}-190 t^{3}+178 t\right) \\
\beta_{\frac{1}{3}}(t)=\frac{1}{19595520}\left(870 t^{8}+9236 t^{7}-7812 t^{6}+7924 t^{5}+7938 t^{4}+7157 t^{3}-5404 t+836\right) \\
\beta_{\frac{2}{3}}(t)=\frac{1}{19595520}\left(1840 t^{8}+12846 t^{7}+14410 t^{6}-10450 t^{5}+14224 t^{4}-7102 t^{3}+13368 t+2784\right) \\
\beta_{\frac{5}{6}}(t)=\frac{1}{19595520}\left(746 t^{8}+3876 t^{7}+2860 t^{6}-2656 t^{5}+4624 t^{4}-3102 t^{3}+3708 t+714\right) \\
\beta_{1}(t)=\frac{1}{19595520}\left(22 t^{8}-96 t^{7}+40 t^{6}-136 t^{5}-64 t^{4}+74 t^{3}-88 t+14\right) \\
\end{gathered}
$$

where $t=\frac{x-x_{n+v_{i}}}{h}$.

We evaluate (9) at $t=0, \frac{1}{6}, 1$ to obtain the discrete one step formula

$$
\begin{aligned}
& y_{n}-10 y_{n+\frac{1}{3}}+20 y_{n+\frac{1}{2}}-15 y_{n+\frac{2}{3}}+4 y_{n+\frac{5}{6}} \\
& =\frac{h^{4}}{19595520}\left[4 f_{n}+2370 f_{n+\frac{1}{6}}+20745 f_{n+\frac{1}{3}}+41920 f_{n+\frac{1}{2}}+10770 f_{n+\frac{2}{3}}-234 f_{n+\frac{5}{6}}+25 f_{n+1}\right] \\
& y_{n+\frac{1}{6}}-4 y_{n+\frac{1}{3}}+6 y_{n+\frac{1}{2}}-4 y_{n+\frac{2}{3}}+y_{n+\frac{5}{6}} \\
& =\frac{h^{4}}{19595520}\left[5 f_{n}-51 f_{n+\frac{1}{6}}+2679 f_{n+\frac{1}{3}}+9854 f_{n+\frac{1}{2}}+2679 f_{n+\frac{2}{3}}-51 f_{n+\frac{5}{6}}+5 f_{n+1}\right] \\
& y_{n+1}-4 y_{n+\frac{5}{6}}+6 y_{n+\frac{2}{3}}-4 y_{n+\frac{1}{2}}+y_{n+\frac{1}{3}}\left[5 f_{n}-30 f_{n+\frac{1}{6}}+54 f_{n+\frac{1}{3}}+2504 f_{n+\frac{1}{2}}+10029 f_{n+\frac{2}{3}}+2574 f_{n+\frac{5}{6}}-16 f_{n+1}\right]
\end{aligned}
$$

The first derivative of $\alpha(t)$ and $\beta(t)$ in (9) gives:

$$
\begin{gathered}
\alpha_{0}^{\prime}(t)=\frac{1}{8}\left(6 t^{2}+2 t+5\right) \alpha_{\frac{1}{3}}^{\prime}(t)=-\frac{1}{4}\left(24 t^{2}+44 t+10\right) \\
\alpha_{\frac{1}{2}}^{\prime}(t)=\frac{1}{2}\left(30 t^{2}+48 t+6\right) \quad \alpha_{\frac{2}{3}}^{\prime}(t)=-\frac{1}{4}\left(96 t^{2}+36 t+7\right) \\
\alpha_{\frac{5}{6}}^{\prime}(t)=\frac{1}{2}\left(9 t^{2}+8 t+1\right) \beta_{\frac{1}{6}}^{\prime}(t)=\frac{1}{19595520}\left(2064 t^{7}+3472 t^{6}-1890 t^{5}+4990 t^{4}+3780 t^{3}-570 t^{2}+178\right)
\end{gathered}
$$




$$
\begin{gathered}
\beta_{\frac{1}{3}}^{\prime}(t)=\frac{1}{19595520}\left(6960 t^{7}+64652 t^{6}-46872 t^{5}+39620 t^{4}+31752 t^{3}+21471 t^{2}-5404\right) \\
\beta_{\frac{1}{2}}^{\prime}(t)=\frac{1}{19595520}\left(14880 t^{7}+12922 t^{6}+86460 t^{5}-52250 t^{4}+56896 t^{3}-21306 t^{2}+13368\right) \\
\beta_{\frac{2}{3}}^{\prime}(t)=\frac{1}{19595520}\left(5968 t^{7}+27132 t^{6}+17160 t^{5}-13280 t^{4}+18496 t^{3}-9306 t^{2}+3708\right) \\
\beta_{\frac{5}{6}}^{\prime}(t)=\frac{1}{19595520}\left(176 t^{7}-672 t^{6}+240 t^{5}-680 t^{4}-256 t^{3}+222 t^{2}-88\right) \\
\beta_{1}^{\prime}(t)=\frac{1}{19595520}\left(40 t^{7}+84 t^{6}-60 t^{5}+40 t^{4}-24 t^{3}-24 t^{2}+14\right)
\end{gathered}
$$

Similarly, the second derivative of $\alpha(t)$ and $\beta(t)$ in (9) gives

$$
\begin{gathered}
\alpha_{0}^{\prime \prime}(t)=\frac{1}{8}(12 t+2) \alpha_{\frac{1}{3}}^{\prime \prime}(t)=-\frac{1}{4}(48 t+44) \\
\alpha_{\frac{1}{2}}^{\prime \prime}(t)=\frac{1}{2}(60 t+48) \alpha_{\frac{2}{3}}^{\prime \prime}(t)=-\frac{1}{4}(192 t+36) \\
\alpha_{\frac{5}{6}}^{\prime \prime}(t)=\frac{1}{2}(18 t+8) \\
\beta_{\frac{1}{3}}^{\prime}(t)=\frac{1}{19595520}\left(672 t^{6}+42 t^{5}-120 t^{4}+40 t^{3}-48 t^{2}+t\right) \\
\beta_{\frac{1}{2}}^{\prime}(t)=\frac{1}{19595520}(t)=\frac{1}{19595520}\left(14448 t^{6}+20832 t^{5}-9450 t^{4}+19960 t^{3}+11340 t^{2}-1140 t\right) \\
\beta_{\frac{2}{3}}^{\prime}(t)=\frac{1}{19595520}\left(41776 t^{6}+162792 t^{5}+85800 t^{4}-53120 t^{3}+55488 t^{2}-18612 t\right) \\
\beta_{\frac{5}{6}}^{\prime}(t)=\frac{1}{19595520}\left(1232 t^{6}-4032 t^{5}+1200 t^{4}-2720 t^{3}-768 t^{2}+444 t\right) \\
\beta_{1}^{\prime}(t)=\frac{1}{19595520}\left(280 t^{6}+516 t^{5}-300 t^{4}+160 t^{3}-72 t^{2}+48 t\right)
\end{gathered}
$$

The third derivative of $\alpha(t)$ and $\beta(t)$ in (9) gives

$$
\begin{gathered}
\alpha_{0}^{\prime \prime \prime}(t)=\frac{1}{8}(12) \alpha_{\frac{1}{3}}^{\prime \prime \prime}(t)=-\frac{1}{4}(48) \\
\alpha_{\frac{1}{2}}^{\prime \prime \prime}(t)=\frac{1}{2}(60) \\
\alpha_{\frac{2}{3}}^{\prime \prime \prime}(t)=-\frac{1}{4}(192)
\end{gathered}
$$




$$
\begin{gathered}
\alpha_{\frac{5}{6}}^{\prime \prime \prime}(t)=\frac{1}{2}(18) \\
\beta_{0}^{\prime \prime \prime}(t)=\frac{1}{19595520}\left(4032 t^{5}+210 t^{4}-480 t^{3}+120 t^{2}-48 t+1\right) \\
\beta_{\frac{1}{6}}^{\prime \prime \prime}(t)=\frac{1}{19595520}\left(86688 t^{5}+104160 t^{4}-37800 t^{3}+59880 t^{2}+22680 t-1140\right) \\
\beta_{\frac{1}{3}}^{\prime \prime \prime}(t)=\frac{1}{19595520}\left(292320 t^{5}+1939560 t^{4}-937440 t^{3}+475440 t^{2}+190512 t+42942\right) \\
\beta_{\frac{1}{2}}^{\prime \prime \prime}(t)=\frac{1}{19595520}\left(624960 t^{5}+387660 t^{4}+1729200 t^{3}-672000 t^{2}+341376 t-42612\right) \\
\beta_{\frac{2}{3}}^{\prime \prime \prime}(t)=\frac{1}{19595520}\left(250656 t^{5}+813960 t^{4}+343200 t^{3}-159360 t^{2}+110976 t-18612\right) \\
\beta_{\frac{5}{6}}^{\prime \prime \prime}(t)=\frac{1}{19595520}\left(7392 t^{5}-20160 t^{4}+4800 t^{3}-8160 t^{2}-1536 t+444\right) \\
\beta_{1}^{\prime \prime \prime}(t)=\frac{1}{19595520}\left(1400 t^{5}+2580 t^{4}-1200 t^{3}+480 t^{2}-144 t+48\right)
\end{gathered}
$$

It is noted that the general fourth order odes involve the first, second and third derivatives. The derivatives can be obtained by imposing that:

$$
y^{\prime}(x)=\frac{1}{h} \sum_{j=0}^{k-1} \alpha_{j}(t) y_{n+j}+\frac{1}{h} \sum_{v_{i}} \alpha_{v_{i}}(t) y_{n+v_{i}}+h^{3}\left(\sum_{j=0}^{k} \beta_{j}(t) f_{n+j}^{\prime}+\sum_{v_{i}} \beta_{v_{i}}(t) f_{n+v_{i}}^{\prime}\right)
$$

By using (14) and evaluating (11), (12) and (13) at $x=x_{n+j}, j=0\left(\frac{1}{6}\right) 1$, we obtain the first, second and the third derivative scheme as follows:

$$
\begin{aligned}
& h y_{n}^{\prime}+47 y_{n+\frac{1}{3}}-114 y_{n+\frac{1}{2}}+93 y_{n+\frac{2}{3}}-26 y_{n+\frac{5}{6}} \\
& =\frac{-h^{4}}{195955200}\left[7658 f_{n}+435930 f_{n+\frac{1}{6}}+1848915 f_{n+\frac{1}{3}}+2845040 f_{n+\frac{1}{2}}+696540 f_{n+\frac{2}{3}}-14418 f_{n+\frac{5}{6}}+1535 f_{n+1}\right] \\
& h y_{n+\frac{1}{6}}^{\prime}+26 y_{n+\frac{1}{3}}-57 y_{n+\frac{1}{2}}+42 y_{n+\frac{2}{3}}-11 y_{n+\frac{5}{6}} \\
& =\frac{-h^{4}}{195955200}\left[235 f_{n}+4713 f_{n+\frac{1}{6}}+454125 f_{n+\frac{1}{3}}+1144150 f_{n+\frac{1}{2}}+291225 f_{n+\frac{2}{3}}-4935 f_{n+\frac{5}{6}}+487 f_{n+1}\right] \\
& h y_{n+\frac{1}{3}}^{\prime}+11 y_{n+\frac{1}{3}}-18 y_{n+\frac{1}{2}}+9 y_{n+\frac{2}{3}}-2 y_{n+\frac{5}{6}} \\
& =\frac{h^{4}}{195955200}\left[7 f_{n}-14 f_{n+\frac{1}{6}}-1000 f_{n+\frac{1}{3}}-17960 f_{n+\frac{1}{2}}-6445 f_{n+\frac{2}{3}}+238 f_{n+\frac{5}{6}}-26 f_{n+1}\right] \\
& h y_{n+\frac{1}{2}}^{\prime}+2 y_{n+\frac{1}{3}}+3 y_{n+\frac{1}{2}}-6 y_{n+\frac{2}{3}}+y_{n+\frac{5}{6}} \\
& =\frac{-h^{4}}{195955200}\left[134 f_{n}-1101 f_{n+\frac{1}{6}}+3960 f_{n+\frac{1}{3}}-49270 f_{n+\frac{1}{2}}-30750 f_{n+\frac{2}{3}}+1611 f_{n+\frac{5}{6}}-184 f_{n+1}\right]
\end{aligned}
$$




$$
\begin{aligned}
& h y_{n+\frac{2}{3}}^{\prime}-y_{n+\frac{1}{3}}+6 y_{n+\frac{1}{2}}-3 y_{n+\frac{2}{3}}-2 y_{n+\frac{5}{6}} \\
& =\frac{h^{4}}{195955200}\left[134 f_{n}-1122 f_{n+\frac{1}{6}}+4425 f_{n+\frac{1}{3}}-35440 f_{n+\frac{1}{2}}-44580 f_{n+\frac{2}{3}}+1146 f_{n+\frac{5}{6}}-163 f_{n+1}\right] \\
& h y_{n+\frac{5}{6}}^{\prime}+2 y_{n+\frac{1}{3}}-9 y_{n+\frac{1}{2}}+18 y_{n+\frac{2}{3}}-11 y_{n+\frac{5}{6}} \\
& =\frac{-h^{4}}{195955200}\left[7 f_{n}-75 f_{n+\frac{1}{6}}+385 f_{n+\frac{1}{3}}-6690 f_{n+\frac{1}{2}}-17715 f_{n+\frac{2}{3}}-1147 f_{n+\frac{5}{6}}+35 f_{n+1}\right] \\
& h y_{n+1}^{\prime}+11 y_{n+\frac{1}{3}}-42 y_{n+\frac{1}{2}}+57 y_{n+\frac{2}{3}}-26 y_{n+\frac{5}{6}} \\
& =\frac{h^{4}}{195955200}\left[235 f_{n}-1158 f_{n+\frac{1}{6}}+283000 f_{n+\frac{1}{2}} 1152375 f_{n+\frac{2}{3}}+449190 f_{n+\frac{5}{6}}+6358 f_{n+1}\right] \\
& h^{2} y_{n}^{\prime \prime}-144 y_{n+\frac{1}{3}}+396 y_{n+\frac{1}{2}}-360 y_{n+\frac{2}{3}}+108 y_{n+\frac{5}{6}} \\
& =\frac{h^{4}}{10886400}\left[19823 f_{n}+322282 f_{n+\frac{1}{6}}+575180 f_{n+\frac{1}{3}}+728600 f_{n+\frac{1}{2}}+140915 f_{n+\frac{2}{3}}+2902 f_{n+\frac{5}{6}}-502 f_{n+1}\right] \\
& h^{2} y_{n+\frac{1}{6}}^{\prime \prime}-108 y_{n+\frac{1}{3}}+288 y_{n+\frac{1}{2}}-252 y_{n+\frac{2}{3}}+72 y_{n+\frac{5}{6}} \\
& =\frac{-h^{4}}{10886400}\left[802 f_{n}-25137 f_{n+\frac{1}{6}}-308500 f_{n+\frac{1}{3}}-442510 f_{n+\frac{1}{2}}-109290 f_{n+\frac{2}{3}}+2983 f_{n+\frac{5}{6}}-348 f_{n+1}\right] \\
& h^{2} y_{n+\frac{1}{3}}^{\prime \prime}-72 y_{n+\frac{1}{3}}+180 y_{n+\frac{1}{2}}-144 y_{n+\frac{2}{3}}+36 y_{n+\frac{5}{6}} \\
& =\frac{h^{4}}{10886400}\left[148 f_{n}-2038 f_{n+\frac{1}{6}}+30285 f_{n+\frac{1}{3}}+196160 f_{n+\frac{1}{2}}+53530 f_{n+\frac{2}{3}}-978 f_{n+\frac{5}{6}}+93 f_{n+1}\right] \\
& h^{2} y_{n+\frac{1}{2}}^{\prime \prime}-36 y_{n+\frac{1}{3}}+72 y_{n+\frac{1}{2}}-36 y_{n+\frac{2}{3}} \\
& =\frac{-h^{4}}{10886400}\left[7 f_{n}-97 f_{n+\frac{1}{6}}+1165 f_{n+\frac{1}{3}}+23050 f_{n+\frac{1}{2}}+1165 f_{n+\frac{2}{3}}-97 f_{n+\frac{5}{6}}+7 f_{n+1}\right] \\
& h^{2} y_{n+\frac{2}{3}}^{\prime \prime}-36 y_{n+\frac{1}{2}}+72 y_{n+\frac{2}{3}}-36 y_{n+\frac{5}{6}} \\
& =\frac{-h^{4}}{10886400}\left[7 f_{n}-42 f_{n+\frac{1}{6}}+50 f_{n+\frac{1}{3}}+920 f_{n+\frac{1}{2}}+23295 f_{n+\frac{2}{3}}+1018 f_{n+\frac{5}{6}}-48 f_{n+1}\right] \\
& h^{2} y_{n+\frac{5}{6}}^{\prime \prime}+36 y_{n+\frac{1}{3}}-144 y_{n+\frac{1}{2}}+180 y_{n+\frac{2}{3}}-72 y_{n+5 / 6} \\
& =\frac{h^{4}}{10886400}\left[148 f_{n}-943 f_{n+\frac{1}{6}}+2130 f_{n+\frac{1}{3}}+48350 f_{n+\frac{1}{2}}+20134 f_{n+\frac{2}{3}}+27177 f_{n+\frac{5}{6}}-1002 f_{n+1}\right] \\
& h^{2} y_{n+1}^{\prime \prime}+72 y_{n+\frac{1}{3}}-252 y_{n+\frac{1}{2}}+288 y_{n+\frac{2}{3}}-108 y_{n+\frac{5}{6}} \\
& =\frac{-h^{4}}{10886400}\left[802 f_{n}-5962 f_{n+\frac{1}{6}}+19825 f_{n+\frac{1}{3}}-137360 f_{n+\frac{1}{2}}-414440 f_{n+\frac{2}{3}}-325342 f_{n+\frac{5}{6}}-19523 f_{n+1}\right]
\end{aligned}
$$




$$
\begin{aligned}
& h^{3} y_{n}^{\prime \prime \prime}+216 y_{n+\frac{1}{3}}-648 y_{n+\frac{1}{2}}+648 y_{n+\frac{2}{3}}-216 y_{n+\frac{5}{6}} \\
& =\frac{-h^{4}}{725760}\left[36799 f_{n}+176608 f_{n+\frac{1}{6}}+55219 f_{n+\frac{1}{3}}+156920 f_{n+\frac{1}{2}}-10459 f_{n+\frac{2}{3}}+9608 f_{n+\frac{5}{6}}-1335 f_{n+1}\right] \\
& h^{3} y_{n+\frac{1}{6}}^{\prime \prime \prime}+216 y_{n+\frac{1}{3}}-648 y_{n+\frac{1}{2}}+648 y_{n+\frac{2}{3}}-216 y_{n+\frac{5}{6}} \\
& =\frac{h^{4}}{725760}\left[1375 f_{n}-46384 f_{n+\frac{1}{6}}-148141 f_{n+\frac{1}{3}}-81912 f_{n+\frac{1}{2}}-29963 f_{n+\frac{2}{3}}+3016 f_{n+\frac{5}{6}}-391 f_{n+1}\right] \\
& h^{3} y_{n+\frac{1}{3}}^{\prime \prime \prime}+216 y_{n+\frac{1}{3}}-648 y_{n+\frac{1}{2}}+648 y_{n+2 / 3}-216 y_{n+5 / 6} \\
& =\frac{-h^{4}}{725760}\left[351 f_{n}-3872 f_{n+\frac{1}{6}}+54163 f_{n+\frac{1}{3}}+114424 f_{n+\frac{1}{2}}+15365 f_{n+\frac{2}{3}}+1160 f_{n+\frac{5}{6}}-151 f_{n+1}\right] \\
& h^{3} y_{n+\frac{1}{2}}^{\prime \prime \prime}+216 y_{n+\frac{1}{3}}-648 y_{n+\frac{1}{2}}+648 y_{n+\frac{2}{3}}-216 y_{n+\frac{5}{6}} \\
& =\frac{h^{4}}{725760}\left[191 f_{n}-1648 f_{n+\frac{1}{6}}+7475 f_{n+\frac{1}{3}}+39416 f_{n+\frac{1}{2}}-28907 f_{n+\frac{2}{3}}+2056 f_{n+\frac{5}{6}}-231 f_{n+1}\right] \\
& h^{3} y_{n+\frac{2}{3}}^{\prime \prime \prime}+216 y_{n+\frac{1}{3}}-648 y_{n+\frac{1}{2}}+648 y_{n+\frac{2}{3}}-216 y_{n+\frac{5}{6}} \\
& =\frac{-h^{4}}{725760}\left[191 f_{n}-1568 f_{n+\frac{1}{6}}+6067 f_{n+\frac{1}{3}}-35592 f_{n+\frac{1}{2}}-32731 f_{n+\frac{2}{3}}+3464 f_{n+\frac{5}{6}}-311 f_{n+1}\right] \\
& h^{3} y_{n+\frac{5}{6}}^{\prime \prime \prime}+216 y_{n+\frac{1}{3}}-648 y_{n+\frac{1}{2}}+648 y_{n+\frac{2}{3}}-216 y_{n+\frac{5}{6}} \\
& =\frac{h^{4}}{725760}\left[351 f_{n}-2608 f_{n+\frac{1}{6}}+8531 f_{n+\frac{1}{3}}-3080 f_{n+\frac{1}{2}}+126709 f_{n+\frac{2}{3}}+46792 f_{n+\frac{5}{6}}-1415 f_{n+1}\right] \\
& h^{3} y_{n+1}^{\prime \prime \prime}+216 y_{n+\frac{1}{3}}-648 y_{n+\frac{1}{2}}+648 y_{n+\frac{2}{3}}-216 y_{n+\frac{5}{6}} \\
& =\frac{-h^{4}}{725760}\left[1375 f_{n}-10016 f_{n+\frac{1}{6}}+31891 f_{n+\frac{1}{3}}-78088 f_{n+\frac{1}{2}}-33787 f_{n+\frac{2}{3}}-177016 f_{n+\frac{5}{6}}-36759 f_{n+1}\right]
\end{aligned}
$$

By combining the schemes (10), the first, second, third derivatives schemes (15) together and write them in block form, using the definition of implicit block method in [9] to obtain the block formula describe as follows:

$$
h^{p} \sum_{j=0}^{q} a_{i, j} y_{n+j}^{\lambda}=h^{\lambda} \sum_{j=0}^{q} e_{i, j} y_{n}^{\lambda}+h^{p-\lambda}\left(\sum_{j+1}^{q} d_{i, j} f_{n}+\sum_{j=1}^{q} b_{i, j} f_{n+j}\right), \quad i=0,1, \cdots, q
$$

$\lambda$ is the power of the derivative of the continuous method and $p$ is the order of the problem to solved: $q=r+s$.

This equation is solved and we obtained values for $y_{n+v_{i}}, y_{n+1}, y_{n+v_{i}}^{\prime}, y_{n+1}^{\prime}, y_{n+v_{i}}^{\prime \prime}, y_{n+1}^{\prime \prime}, y_{n+v_{i}}^{\prime \prime \prime}$ and $y_{n+1}^{\prime \prime \prime}$ as follows:

$$
\begin{aligned}
y_{n+\frac{1}{6}}= & y_{n}+\frac{1}{6} h y_{n}^{\prime}+\frac{1}{72} h^{2} y_{n}^{\prime \prime}+\frac{1}{1296} h^{3} y_{n}^{\prime \prime \prime}+\frac{h^{4}}{4702924800}\left[95929 f_{n}+112028 f_{n+\frac{1}{6}}\right. \\
& \left.-115165 f_{n+\frac{1}{3}}+97320 f_{n+\frac{1}{2}}-53465 f_{n+\frac{2}{3}}+16876 f_{n+\frac{5}{6}}-2323 f_{n+1}\right]
\end{aligned}
$$




$$
\begin{aligned}
& y_{n+\frac{1}{3}}=y_{n}+\frac{1}{3} h y_{n}^{\prime}+\frac{1}{18} h^{2} y_{n}^{\prime \prime}+\frac{1}{162} h^{3} y_{n}^{\prime \prime \prime}+\frac{h^{4}}{18370800}\left[4127 f_{n}+8782 f_{n+\frac{1}{6}}\right. \\
& \left.-6965 f_{n+\frac{1}{3}}+5820 f_{n+\frac{1}{2}}-3175 f_{n+\frac{2}{3}}+998 f_{n+\frac{5}{6}}-137 f_{n+1}\right] \\
& y_{n+\frac{1}{2}}=y_{n}+\frac{1}{2} h y_{n}^{\prime}+\frac{1}{8} h^{2} y_{n}^{\prime \prime}+\frac{1}{48} h^{3} y_{n}^{\prime \prime \prime}+\frac{h^{4}}{6451200}\left[5471 f_{n}+15228 f_{n+\frac{1}{6}}\right. \\
& \left.-8775 f_{n+\frac{1}{3}}+8120 f_{n+\frac{1}{2}}-4455 f_{n+\frac{2}{3}}+14040 f_{n+\frac{5}{6}}-193 f_{n+1}\right] \\
& y_{n+\frac{2}{3}}=y_{n}+\frac{2}{3} h y_{n}^{\prime}+\frac{2}{9} h^{2} y_{n}^{\prime \prime}+\frac{4}{81} h^{3} y_{n}^{\prime \prime \prime}+\frac{h^{4}}{1148175}\left[1220 f_{n}+3904 f_{n+\frac{1}{6}}\right. \\
& \left.-1580 f_{n+\frac{1}{3}}+1920 f_{n+\frac{1}{2}}-1015 f_{n+\frac{2}{3}}+320 f_{n+\frac{5}{6}}-44 f_{n+1}\right] \\
& y_{n+\frac{5}{6}}=y_{n}+\frac{5}{6} h y_{n}^{\prime}+\frac{25}{72} h^{2} y_{n}^{\prime \prime}+\frac{125}{1296} h^{3} y_{n}^{\prime \prime \prime}+\frac{h^{4}}{188116992}\left[807125 f_{n}+2807500 f_{n+\frac{1}{6}}\right. \\
& \left.-790625 f_{n+\frac{1}{3}}+1425000 f_{n+\frac{1}{2}}-653125 f_{n+\frac{2}{3}}+214256 f_{n+\frac{5}{6}}-29375 f_{n+1}\right] \\
& y_{n+1}=y_{n}+h y_{n}^{\prime}+\frac{1}{2} h^{2} y_{n}^{\prime \prime}+\frac{1}{6} h^{3} y_{n}^{\prime \prime \prime}+\frac{h^{4}}{25200}\left[191 f_{n}+702 f_{n+\frac{1}{6}}\right. \\
& \left.-135 f_{n+\frac{1}{3}}+380 f_{n+\frac{1}{2}}-135 f_{n+\frac{2}{3}}+54 f_{n+\frac{5}{6}}-7 f_{n+1}\right] \\
& y_{n+\frac{1}{6}}^{\prime}=h y_{n}^{\prime}+\frac{1}{6} h^{2} y_{n}^{\prime \prime}+\frac{1}{72} h^{3} y_{n}^{\prime \prime \prime}+\frac{h^{3}}{26123963443200}\left[11458543529 f_{n}+1688294016 f_{n+\frac{1}{6}}-16488356235 f_{n+\frac{1}{3}}\right. \\
& \left.+13670222640 f_{n+\frac{1}{2}}+7552518045 f_{n+\frac{2}{3}}+2378490756 f_{n+\frac{5}{6}}-326924161 f_{n+1}\right] \\
& y_{n+\frac{1}{3}}^{\prime}=h y_{n}^{\prime}+\frac{1}{3} h^{2} y_{n}^{\prime \prime}+\frac{1}{18} h^{3} y_{n}^{\prime \prime \prime}+\frac{h^{3}}{6123600}\left[13774 f_{n}+35976 f_{n+\frac{1}{6}}\right. \\
& \left.-24465 f_{n+\frac{1}{3}}+20800 f_{n+\frac{1}{2}}-11370 f_{n+\frac{2}{3}}+3576 f_{n+\frac{5}{6}}-491 f_{n+1}\right] \\
& y_{n+\frac{1}{2}}^{\prime}=h y_{n}^{\prime}+\frac{1}{2} h^{2} y_{n}^{\prime \prime}+\frac{1}{8} h^{3} y_{n}^{\prime \prime \prime}+\frac{h^{3}}{1075200}\left[5877 f_{n}+19188 f_{n+\frac{1}{6}}\right. \\
& \left.-8055 f_{n+\frac{1}{3}}+8960 f_{n+\frac{1}{2}}-4905 f_{n+\frac{2}{3}}+1548 f_{n+\frac{5}{6}}-213 f_{n+1}\right] \\
& y_{n+\frac{2}{3}}^{\prime}=h y_{n}^{\prime}+\frac{2}{3} h^{2} y_{n}^{\prime \prime}+\frac{2}{9} h^{3} y_{n}^{\prime \prime \prime}+\frac{h^{3}}{382725}\left[3863 f_{n}+13992 f_{n+\frac{1}{6}}\right. \\
& \left.-3390 f_{n+\frac{1}{3}}+6800 f_{n+\frac{1}{2}}-3255 f_{n+\frac{2}{3}}+1032 f_{n+\frac{5}{6}}-142 f_{n+1}\right]
\end{aligned}
$$




$$
\begin{aligned}
& y_{n+5 / 6}^{\prime}=h y_{n}^{\prime}+\frac{5}{6} h^{2} y_{n}^{\prime \prime}+\frac{25}{72} h^{3} y_{n}^{\prime \prime \prime}+\frac{h^{3}}{31352832}\left[505625 f_{n}+1945500 f_{n+\frac{1}{6}}-256875 f_{n+\frac{1}{3}}\right. \\
& \left.+1070000 f_{n+\frac{1}{2}}-358125 f_{n+\frac{2}{3}}+136500 f_{n+\frac{5}{6}}-18625 f_{n+1}\right] \\
& y_{n+1}^{\prime}=h y_{n}^{\prime}+h^{2} y_{n}^{\prime \prime}+\frac{1}{2} h^{3} y_{n}^{\prime \prime \prime}+\frac{h^{3}}{8400}\left[198 f_{n}+792 f_{n+\frac{1}{6}}-45 f_{n+\frac{1}{3}}+480 f_{n+\frac{1}{2}}-90 f_{n+\frac{2}{3}}+72 f_{n+\frac{5}{6}}-7 f_{n+1}\right] \\
& y_{n+\frac{1}{6}}^{\prime \prime}=h^{2} y_{n}^{\prime \prime}+\frac{1}{6} h^{3} y_{n}^{\prime \prime \prime} \\
& +\frac{h^{2}}{4354560}\left[28549 f_{n}+57750 f_{n+\frac{1}{6}}-51453 f_{n+\frac{1}{3}}+42484 f_{n+\frac{1}{2}}-23109 f_{n+\frac{2}{3}}+7254 f_{n+\frac{5}{6}}-995 f_{n+1}\right] \\
& y_{n+\frac{1}{3}}^{\prime \prime}=h^{2} y_{n}^{\prime \prime}+\frac{1}{3} h^{3} y_{n}^{\prime \prime \prime}+\frac{h^{2}}{68040}\left[1027 f_{n}+3492 f_{n+\frac{1}{6}}-1680 f_{n+\frac{1}{3}}+1576 f_{n+\frac{1}{2}}-873 f_{n+\frac{2}{3}}+276 f_{n+\frac{5}{6}}-38 f_{n+1}\right] \\
& y_{n+\frac{1}{2}}^{\prime \prime}=h^{2} y_{n}^{\prime \prime}+\frac{1}{2} h^{3} y_{n}^{\prime \prime \prime}+\frac{h^{2}}{53760}\left[1265 f_{n}+4950 f_{n+\frac{1}{6}}-801 f_{n+\frac{1}{3}}+2100 f_{n+\frac{1}{2}}-1089 f_{n+\frac{2}{3}}+342 f_{n+\frac{5}{6}}-47 f_{n+1}\right] \\
& y_{n+\frac{2}{3}}^{\prime \prime}=h^{2} y_{n}^{\prime \prime}+\frac{2}{3} h^{3} y_{n}^{\prime \prime \prime}+\frac{h^{2}}{8505}\left[272 f_{n}+1128 f_{n+\frac{1}{6}}-18 f_{n+\frac{1}{3}}+656 f_{n+\frac{1}{2}}-210 f_{n+\frac{2}{3}}+72 f_{n+\frac{5}{6}}-10 f_{n+1}\right] \\
& y_{n+\frac{5}{6}}^{\prime \prime}=h^{2} y_{n}^{\prime \prime}+\frac{5}{6} h^{3} y_{n}^{\prime \prime \prime}+\frac{h^{2}}{870912}\left[1409 f_{n}+6030 f_{n+\frac{1}{6}}-375 f_{n+\frac{1}{3}}+4100 f_{n+\frac{1}{2}}-225 f_{n+\frac{2}{3}}+462 f_{n+\frac{5}{6}}-55 f_{n+1}\right] \\
& y_{n+1}^{\prime \prime}=h^{2} y_{n}^{\prime \prime}+h^{3} y_{n}^{\prime \prime \prime}+\frac{h^{2}}{840}\left[41 f_{n}+180 f_{n+\frac{1}{6}}+18 f_{n+\frac{1}{3}}+136 f_{n+\frac{1}{2}}+9 f_{n+\frac{2}{3}}+36 f_{n+\frac{5}{6}}\right] \\
& y_{n+\frac{1}{6}}^{\prime \prime \prime}=h^{3} y_{n}^{\prime \prime \prime}+\frac{h}{362880}\left[19087 f_{n}+65112 f_{n+\frac{1}{6}}-46461 f_{n+\frac{1}{3}}+37504 f_{n+\frac{1}{2}}-20211 f_{n+\frac{2}{3}}+6312 f_{n+\frac{5}{6}}-863 f_{n+1}\right] \\
& y_{n+\frac{1}{3}}^{\prime \prime \prime}=h^{3} y_{n}^{\prime \prime \prime}+\frac{h}{22680}\left[1139 f_{n}+5640 f_{n+\frac{1}{6}}+33 f_{n+\frac{1}{3}}+1328 f_{n+\frac{1}{2}}-807 f_{n+\frac{2}{3}}+264 f_{n+\frac{5}{6}}-37 f_{n+1}\right] \\
& y_{n+\frac{1}{2}}^{\prime \prime \prime}=h^{3} y_{n}^{\prime \prime \prime}+\frac{h}{13440}\left[685 f_{n}+3240 f_{n+\frac{1}{6}}+561 f_{n+\frac{1}{3}}+2176 f_{n+\frac{1}{2}}-729 f_{n+\frac{2}{3}}+216 f_{n+\frac{5}{6}}-29 f_{n+1}\right] \\
& y_{n+\frac{2}{3}}^{\prime \prime \prime}=h^{3} y_{n}^{\prime \prime \prime}+\frac{h}{45360}\left[2288 f_{n}+11136 f_{n+\frac{1}{6}}+3072 f_{n+\frac{1}{3}}+7583 f_{n+\frac{1}{2}}+1392 f_{n+\frac{2}{3}}+384 f_{n+\frac{5}{6}}-64 f_{n+1}\right] \\
& y_{n+\frac{5}{6}}^{\prime \prime \prime}=h^{3} y_{n}^{\prime \prime \prime}+\frac{h}{72576}\left[3715 f_{n}+17400 f_{n+\frac{1}{6}}+6375 f_{n+\frac{1}{3}}+15384 f_{n+\frac{1}{2}}+11625 f_{n+\frac{2}{3}}+5640 f_{n+\frac{5}{6}}-275 f_{n+1}\right] \\
& y_{n+1}^{\prime \prime \prime}=h^{3} y_{n}^{\prime \prime \prime}+\frac{h}{840}\left[41 f_{n}+216 f_{n+\frac{1}{6}}+27 f_{n+\frac{1}{3}}+272 f_{n+\frac{1}{2}}+27 f_{n+\frac{2}{3}}+216 f_{n+\frac{5}{6}}+41 f_{n+1}\right]
\end{aligned}
$$

\section{Analysis of the Properties of the Block}

In this section, we carry out the analysis of the basic properties of the new method. 


\subsection{Order of the Method}

\subsubsection{Order of the Block (17)}

The linear operator of the block (17) is defined as:

$$
L\{y(x): h\}=Y_{m}-e y_{m}+h^{\mu-\lambda} d f\left(y_{m}\right)+h^{\mu-\lambda} b F\left(y_{m}\right)
$$

By expanding $y\left(x_{n}+i h\right)$ and $f\left(x_{n}+j h\right)$ in Taylor series, (21) becomes:

$$
L\{y(x): h\}=C_{0} y(x)+C_{1} h y^{\prime}(x)+C_{2} h^{2} y^{\prime \prime}(x)+\cdots+C_{p} h^{p} y^{(p)}(x)
$$

The block (17) and associated linear operator are said to have order $p$ if

$$
C_{0}=C_{1}=\cdots=C_{p+3}=0, C_{p+4} \neq 0 . \text { See [10]. }
$$

The term $C_{p+4}$ is called the error constant and implies that the local truncation error is given by:

$$
t_{n+k}=C_{p+4} h^{(p+4)} y^{(p+4)}\left(x_{n}\right)+0 h^{(p+5)}
$$

Hence the block (17) has order 7 with error constant:

$$
C_{p+4}=\left[\begin{array}{ccc}
\frac{15739}{10861273143705600} & \frac{733}{33941478574080} & \frac{1}{11496038400} \\
\frac{37}{165729875850} & \frac{198125}{434450925748224} & \frac{1}{1231718400}
\end{array}\right]^{\mathrm{T}}
$$

\subsubsection{Order and Error Constant of the Main Method (10c)}

By rewriting the main method (10c) in the form:

$$
\begin{aligned}
& y_{n+1}-4 y_{n+\frac{5}{6}}+6 y_{n+\frac{2}{3}}-4 y_{n+\frac{1}{2}}+y_{n+\frac{1}{3}} \\
& -\frac{h^{4}}{19595520}\left[5 f_{n}-30 f_{n+\frac{1}{6}}+54 f_{n+\frac{1}{3}}+2504 f_{n+\frac{1}{2}}+10029 f_{n+\frac{2}{3}}+2574 f_{n+\frac{5}{6}}-16 f_{n+1}\right]=0
\end{aligned}
$$

Expanding (24) in Taylor series in the form:

$$
\begin{aligned}
& \sum_{j=0}^{\infty} \frac{h^{j}}{j !} y_{n}^{(j)}-4 \sum_{j=0}^{\infty}\left(\frac{5}{6}\right)^{j} \frac{h^{j}}{j !} y_{n}^{(j)}+6 \sum_{j=0}^{\infty}\left(\frac{2}{3}\right)^{j} \frac{h^{j}}{j !} y_{n}^{(j)}-4 \sum_{j=0}^{\infty}\left(\frac{1}{2}\right)^{j} \frac{h^{j}}{j !} y_{n}^{(j)}+\sum_{j=0}^{\infty}\left(\frac{1}{3}\right)^{j} \frac{h^{j}}{j !} y_{n}^{(j)} \\
& -\sum_{j=0}^{\infty} \frac{h^{(j+4)}}{j !} y_{n}^{(j+4)}\left[\frac{-30}{19595520}\left(\frac{1}{6}\right)^{j}+\frac{54}{19595520}\left(\frac{1}{3}\right)^{j}+\frac{2504}{19595520}\left(\frac{1}{2}\right)^{j}\right. \\
& \left.+\frac{10029}{19595520}\left(\frac{2}{3}\right)^{j}+\frac{2574}{19595520}\left(\frac{5}{6}\right)^{j}-\frac{16}{19595520}\right]-\frac{5}{19595520} h^{4} y_{n}^{(4)}=0
\end{aligned}
$$

Since $C_{0}, \cdots, C_{10}=0$ but $C_{11} \neq 0$ see [10]; then the main scheme is of order 7 and the error constant is:

$$
C_{p+4}=\frac{1}{1097098297344} \text {. }
$$

\subsection{Zero Stability of the Block}

The block (17) is said to be Zero stable if the roots $Z_{\mathrm{s}}=1,2, \cdots, N$ of the characteristic polynomial $\rho(z)=\operatorname{det}(z A-E)$, satisfies $|z| \leq 1$ and the root $|z|=1$ has multiplicity not exceeding the order of the differential equation. Moreover as

$$
h^{\mu} \rightarrow 0, \rho(z)=z^{r-\mu}(\lambda-1),
$$

where $\mu$ is the order of the differential equation, for the block (19), $r=10, \mu=4$ 


$$
\rho(z)=\lambda^{5}(\lambda-1)=0 \Rightarrow \lambda=0,0,0,0,0,1
$$

Hence our method is Zero stable.

\subsection{Consistency of the Main Method (10c)}

From main method (10c), the first and second characteristics polynomials of the method are given by:

$$
\rho(r)=r-4 r^{\frac{5}{6}}+6 r^{\frac{2}{3}}-4 r^{\frac{1}{2}}+r^{\frac{1}{3}}
$$

and

$$
\begin{aligned}
\sigma(r)= & \frac{5}{19595520}-\frac{30}{19595520} r^{\frac{1}{6}}+\frac{54}{19595520} r^{\frac{2}{3}}+\frac{2504}{19595520} r^{\frac{1}{2}} \\
& +\frac{10029}{19595520} r^{\frac{1}{3}}+\frac{2574}{19595520} r^{\frac{5}{6}}-\frac{16}{19595520} r
\end{aligned}
$$

the method (10c) is consistent since it satisfies the following conditions:

1. The order of the method is $p=7 \geq 1$ which is obvious.

2. For the method $\alpha_{1}=1, \alpha_{\frac{5}{6}}=-4, \alpha_{\frac{2}{3}}=6, \alpha_{\frac{1}{2}}=-4$ and $\alpha_{\frac{1}{3}}=1$, thus

$$
\sum_{j} \alpha_{j}=1-4+6-4+1=0, j=\frac{1}{3}\left(\frac{1}{6}\right) 1 .
$$

3. $\rho(r)=r-4 r^{\frac{5}{6}}+6 r^{\frac{2}{3}}-4 r^{\frac{1}{2}}+r^{\frac{1}{3}}$.

4. it follows from here that $\rho(1)=0=\rho^{\prime}(1)$ showing that the condition (3) is satisfied as well.

5. Note that:

$$
\begin{gathered}
\rho^{(i v)}(r)=\frac{455}{324} r^{-\frac{13}{6}}-\frac{112}{27} r^{-\frac{10}{3}}+\frac{15}{4} r^{-\frac{7}{2}}-\frac{80}{81} r^{-\frac{11}{3}} \\
\Rightarrow \rho^{(i v)}(1)=4 ! \sigma(1) .
\end{gathered}
$$

For the principal root $r=1$ : it is observed that the last condition above is satisfied, hence the main method is consistent.

\subsection{Convergence}

The necessary and sufficient condition for a numerical method to be convergent is for it to be consistent and Zero stable. Thus since it has been successfully shown from the above condition, it could be seen that our method is convergent.

\subsection{Region of Absolute Stability of the Method}

We consider the stability polynomial written in general form:

$$
\pi(r, \bar{h})=\rho(r)-\bar{h} \sigma(r)=0
$$

where $\bar{h}=h^{2} \lambda$ and $\lambda=\frac{d f}{\delta y}$ is assumed constant. The stability polynomial of the main method (10c) becomes:

$$
\begin{aligned}
& \left(r-4 r^{\frac{5}{6}}+6 r^{\frac{2}{3}}-4 r^{\frac{1}{2}}+r^{\frac{1}{3}}\right)-\bar{h}\left(\frac{5}{19595520} r^{0}-\frac{30}{19595520} r^{\frac{1}{6}}+\frac{54}{19595520} r^{\frac{1}{3}}\right. \\
& \left.+\frac{2504}{19595520} r^{\frac{1}{2}}+\frac{10029}{19595520} r^{\frac{2}{3}}+\frac{2574}{19595520} r^{\frac{5}{6}}-\frac{16}{19595520} r\right)=0
\end{aligned}
$$


Adopting the boundary locus method whose equation is given by:

$$
\bar{h}=\frac{\rho(r)}{\sigma(r)}
$$

By inserting the values of $\rho(r)$ and $\sigma(r)$ into (27) and evaluate, we obtain the following results as displayed in the table below:

\begin{tabular}{cccccccc}
\hline$\theta$ & $0^{\circ}$ & $30^{\circ}$ & $60^{\circ}$ & $90^{\circ}$ & $120^{\circ}$ & $150^{\circ}$ & $180^{\circ}$ \\
\hline $\bar{h}(\theta)$ & 0 & -0.075 & -1.203 & -6.088 & -19.241 & -46.976 & -96.407
\end{tabular}

From here, it could be seen that the region of absolute stability of the method is given by $x(\theta)=(-96.407,0)$ which satisfies the condition for A-stability, similarly the interval of periodicity lies in interval $x(\theta)=(-\infty, 0)$.

\section{Numerical Experiments}

To test the accuracy, workability and suitability of the method, I adopted our method to solving some initial value problems of fourth order ordinary differential equations.

\section{Test Problem 1}

I consider special fourth order problem:

$$
\begin{aligned}
& y^{i v}=x \\
& y(0)=0, y^{\prime}(0)=1, y^{\prime \prime}(0)=0, y^{\prime \prime \prime}(0)=0, h=0.1
\end{aligned}
$$

Whose exact solution is:

$$
y(x)=\frac{x^{5}}{120}+x
$$

My method was used to solve the problem and result compared with [6]. The result is as shown in Table 1.

\section{Test Problem 2}

I consider a linear fourth order problem

$$
\begin{gathered}
y^{i v}+y^{\prime \prime}=0,0 \leq x \leq \frac{\pi}{2} \\
y(0)=0, y^{\prime}(0)=\frac{1.1}{72-50 \pi}, y^{\prime \prime}(0)=\frac{1}{144-100 \pi}, y^{\prime \prime \prime}=\frac{1.2}{144-100 \pi}, h=\frac{0.1}{32}
\end{gathered}
$$

Whose exact solution is given by:

$$
y(x)=\frac{1-x-\cos x-1.2 \sin x}{144-100 \pi}
$$

My method was used to solve the problem and result compared with [8]. The result is as shown in Table 2.

\section{Numerical Results}

I make use of the following Notations in the table of results:

XVAL: Value of the independent variable where numerical value is taken.

ERC: Exact result at XVAL.

NRC: Our Numerical result at XVAL.

ERR: Error of our result at XVAL.

\section{Discussion of Results}

In this paper, I propose an accurate five off-step points modified implicit block algorithm for the numerical solution of initial value problems of fourth order ordinary differential equations. For better performance of the method, step size is chosen within the stability interval. 
Table 1. Showing results for problem 1.

\begin{tabular}{|c|c|c|c|c|}
\hline XVAL & ERC & NRC & ERR P = $7 \mathrm{~K}=1$ & ERR in [6] $P=4 K=6$ \\
\hline 0.1 & 0.1000000833333340 & 0.10000008333349980 & $1.658 \mathrm{E}-13$ & $7.000 \mathrm{E}-10$ \\
\hline 0.2 & 0.20000266666666690 & 0.20000266666998294 & $3.316 \mathrm{E}-12$ & $8.999 \mathrm{E}-10$ \\
\hline 0.3 & 0.300020250000000004 & 0.30002025000718312 & $7.183 \mathrm{E}-12$ & $2.999 \mathrm{E}-09$ \\
\hline 0.4 & 0.400008533333333333 & 0.40000853339982528 & $6.649 \mathrm{E}-11$ & $5.100 \mathrm{E}-09$ \\
\hline 0.5 & 0.500260416666666665 & 0.50026041667657280 & $9.906 \mathrm{E}-11$ & $7.799 \mathrm{E}-09$ \\
\hline 0.6 & 0.600648000000000007 & 0.60064800003216824 & $3.217 \mathrm{E}-11$ & $1.180 \mathrm{E}-08$ \\
\hline 0.7 & 0.701400583333333344 & 0.70140058343576487 & $2.432 \mathrm{E}-10$ & $1.240 \mathrm{E}-08$ \\
\hline 0.8 & 0.802730666666666670 & 0.80273066698686870 & $3.202 \mathrm{E}-10$ & $1.410 \mathrm{E}-08$ \\
\hline 0.9 & 0.904920750000000005 & 0.90492075025408587 & $2.540 \mathrm{E}-10$ & $1.880 \mathrm{E}-08$ \\
\hline 1.0 & 1.00833333333333300 & 1.00833333359573400 & $2.024 \mathrm{E}-10$ & $2.600 \mathrm{E}-08$ \\
\hline
\end{tabular}

Table 2. Showing results for problem 2.

\begin{tabular}{cccccc}
\hline XVAL & ERC & NRC & ERR P $=7$ K = 1 & ERR in $[8] \mathbf{P}=\mathbf{6}$ K $=\mathbf{4}$ \\
\hline 0.103150 & 0.001300799589367158 & 0.001300799589367196 & $0.38142683 \mathrm{E}-18$ & $0.49873299 \mathrm{E}-15$ \\
0.206250 & 0.002531773700195635 & 0.002531773700195672 & $0.37184370 \mathrm{E}-17$ & $0.67654215 \mathrm{E} 15$ \\
0.306250 & 0.003652478978884993 & 0.003652478978887675 & $0.26822346 \mathrm{E}-16$ & $0.31350790 \mathrm{E}-14$ \\
0.406250 & 0.004695953223180484 & 0.004695953223180513 & $0.29384802 \mathrm{E}-16$ & $0.94360283 \mathrm{E}-13$ \\
0.506250 & 0.005657642360803446 & 0.005657642360803864 & $0.41813224 \mathrm{E}-15$ & $0.22116856 \mathrm{E}-13$ \\
0.603125 & 0.006507754608034524 & 0.00650775460803811 & $0.38734880 \mathrm{E}-15$ & $0.43379362 \mathrm{E} 13$ \\
0.703125 & 0.007298314767638522 & 0.007298314767638809 & $0.28714827 \mathrm{E}-15$ & $0.77870869 \mathrm{E}-13$ \\
0.803125 & 0.007998520222728983 & 0.007998520222737657 & $0.86740034 \mathrm{E}-14$ & $0.12863494 \mathrm{E} 12$ \\
0.903125 & 0.008607246703302495 & 0.008607246703309575 & $0.70802448 \mathrm{E}-14$ & $0.19927115 \mathrm{E}-12$ \\
1.003125 & 0.009124283967030094 & 0.009124283967034006 & $0.35121472 \mathrm{E}-14$ & $0.29323245 \mathrm{E} 12$ \\
\hline
\end{tabular}

The order of my method is of order 7 higher than that of [6] of order 4, which collaborates the principle, that the higher the order of a method is, the more accurate it is. The absolute errors in [6] are more than those of the new methods; this also means that the new methods are accurate than [6] which is of order 4 and implemented in block mode.

The results of my new method when also compared with the block method proposed by [8] showed that my method is more accurate.

\section{References}

[1] Lambert, J.D. (1973) Computational Methods in ODEs. John Wiley \& Sons, New York.

[2] Fatunla, S.O (1991) Block Method for Second Order IVPs. International Journal of Computer Mathematics, 41, 55-63. http://dx.doi.org/10.1080/00207169108804026

[3] Brujnano, L. and Trigiante, D. (1998) Solving Differential Problems by Multistep Initial and Boundary Value Methods. Amsterdam, Gordon and Breach Science Publishers, Netherlands.

[4] Vigo-Aguiar, J. and Ramos, H. (2006) Variable Step Size Implementation of Multistep Method for $y^{\prime \prime}=f\left(x, y, y^{\prime}\right)$. Journal of Computational and Applied Mathematics, 192, 114-131. http://dx.doi.org/10.1016/j.cam.2005.04.043

[5] Omar, Z. (1999) Developing Parallel Block Method for Solving Higher Orders ODES Directly. PhD Thesis, University 
Putra, Malaysia.

[6] Mohammed, U. (2010) A Six Step Block Method for Solution of Fourth Order Ordinary Differential Equations. The Pacific Journal of Science and Technology, 11, 258-265

[7] Ademiluyi, R.A, Duromola, M.K. and Bolaji, B. (2014) Modified Block Method for the Direct Solution of Initial Value Problems of Fourth Order Ordinary Differential Equations. Australian Journal of Basic and Applied Sciences, 8, 389-394.

[8] Kayode, S.J. (2008) A Zero Stable Method for Direct Solution of Fourth Order Ordinary Differential Equation. American Journal of Applied Sciences, 5, 1461-1466. http://dx.doi.org/10.3844/ajassp.2008.1461.1466

[9] Shampine, L.F. and Watts, H.A. (1969) Block Implicit One Step Methods. Mathematics of Computation, 23, 731-740. http://dx.doi.org/10.1090/S0025-5718-1969-0264854-5

[10] Badmus, A.M. and Yahaya, Y.A. (2014) New Algorithm of Obtaining Order and Error Constants of Third Order Linear Multistep Method. Asian Journal of Fuzzy and Applied Mathematics, 2, 190-194. 